\title{
CD10 expression in peripheral T-cell lymphomas complicated by a proliferation of large B-cells
}

Kaaren K Reichard ${ }^{1}$, Erich J Schwartz ${ }^{1}$, John P Higgins ${ }^{1}$, Balasubramanian Narasimhan ${ }^{2}$, Roger A Warnke ${ }^{1}$ and Yasodha Natkunam ${ }^{1}$

${ }^{1}$ Department of Pathology, Stanford University Medical Center, Stanford, CA, USA and ${ }^{2}$ Health Research and Policy, Stanford University Medical Center, Stanford, CA, USA

CD10 expression by the neoplastic $T$ cells in angioimmunoblastic T-cell lymphoma was recently described. As cases of peripheral T-cell lymphoma, unspecified, fail to show similar CD10 expression, this feature helps discriminate between these two entities, particularly in cases exhibiting morphologic overlap. Given these findings, we studied CD10 expression in a subtype of peripheral T-cell lymphoma known as peripheral T-cell lymphoma complicated by a proliferation of large B cells and compared it with angioimmunoblastic T-cell lymphoma and angioimmunoblastic T-cell lymphoma with a large B-cell proliferation. A total of 33 cases were identified including peripheral T-cell lymphoma complicated by a proliferation of large B cells (10), angioimmunoblastic T-cell lymphoma (10) and angioimmunoblastic T-cell lymphoma with a large B-cell proliferation (13). Diagnoses were established by hematoxylin and eosin (H\&E) stain, immunohistochemistry and/or molecular findings (polymerase chain reaction for T-cell receptor-gamma gene rearrangement). Two of 10 cases of peripheral T-cell lymphoma complicated by a proliferation of large B cells showed aberrant CD10 expression (20\%) compared to 9/10 cases of angioimmunoblastic T-cell lymphoma $(90 \%)$ and $8 / 13$ of angioimmunoblastic T-cell lymphoma with a large B-cell proliferation (62\%). One case each of angioimmunoblastic T-cell lymphoma and angioimmunoblastic T-cell lymphoma with a large B-cell proliferation showed a rare, but not unequivocal, $\mathrm{CD} 10(+)$ atypical cell. Four cases of angioimmunoblastic T-cell lymphoma with a large B-cell proliferation were CD10 negative. Of the $2 \mathrm{CD} 10(+)$ peripheral T-cell lymphoma complicated by a proliferation of large B cells, one had no H\&E or IHC features of angioimmunoblastic T-cell lymphoma and showed only a rare positive cell. The second case, a lung biopsy, exhibited diffuse CD10 tumor cell positivity. The predominant staining pattern in the $\mathrm{CD} 10(+)$ cases was characterized by scattered, mostly individual, morphologically neoplastic cells. A rare case showed clusters of positive cells. Our data indicate that only $20 \%$ of cases of peripheral T-cell lymphoma complicated by a proliferation of large B cells show CD10 expression by the neoplastic $T$ cells in contrast to angioimmunoblastic T-cell lymphoma and angioimmunoblastic T-cell lymphoma with a large B-cell proliferation which exhibit CD10 staining in 90 and $62 \%$ of cases, respectively. This finding does not reach statistical significance with a $P$-value of 0.57 (Fisher's exact test). As these entities appear to be biologically distinct and may portend different overall survivals, CD10 expression may serve as an additional discriminating criterion.

Modern Pathology (2006) 19, 337-343. doi:10.1038/modpathol.3800536; published online 6 January 2006

Keywords: T cell; lymphoma; CD10; large B cell; angioimmunoblastic; EBV

Peripheral T-cell lymphomas are generally associated with an overall poor prognosis and aggressive disease course. In the current World Health Organization classification of hematolymphoid neoplasms,

Correspondence: Dr KK Reichard, MD, Department of Pathology, 1 University of New Mexico, MSC-08-4640, Albuquerque, NM 87131, USA.

E-mail: kreichard@salud.unm.edu

Received 2 September 2005; revised 3 November 2005; accepted 5 November 2005; published online 6 January 2006 only a few subtypes within the heterogeneous category of peripheral T-cell lymphoma have been recognized. ${ }^{1}$ They are important to recognize for biologic, therapeutic and prognostic reasons. For example, angioimmunoblastic T-cell lymphoma is typically associated with systemic symptoms including generalized lymphadenopathy, hepatosplenomegaly, polyclonal hypergammaglobulinemia, may show an initial response to steroid therapy, and portends an average survival of 3 years.

Cases of angioimmunoblastic T-cell lymphoma may be accompanied by large B cells which are often 
positive for Epstein-Barr virus RNA. ${ }^{2}$ In such cases the $\mathrm{B}$ cells are typically scattered individually throughout the neoplasm and are highlighted by a CD20 immunohistochemical stain. A similar finding, although with a dramatic increase in the number of large B cells, has been reported in cases of peripheral T-cell lymphoma, unspecified, and is termed peripheral T-cell lymphoma complicated by a proliferation of large B cells. ${ }^{3}$ Such a proliferation has also been reported in angioimmunoblastic T-cell lymphoma. ${ }^{4}$

Recently, expression of CD10 by the neoplastic T cells in angioimmunoblastic T-cell lymphoma was reported. ${ }^{5}$ As cases of peripheral T-cell lymphoma, unspecified, failed to show a similar pattern of aberrant CD10 expression, CD10 emerged as a useful tool to help discriminate peripheral T-cell lymphoma from angioimmunoblastic T-cell lymphoma. The expression of CD10 is particularly useful in cases which show hybrid morphologic and/or immunohistochemical features of both angioimmunoblastic T-cell lymphoma and peripheral T-cell lymphoma.

In the context of these findings, we chose to examine the CD10 expression pattern in the recently described subtype of peripheral T-cell lymphoma, peripheral T-cell lymphoma complicated by a proliferation of large B cells, as compared to angioimmunoblastic T-cell lymphoma with a large B-cell proliferation. The goal of the study was to determine if aberrant CD10 expression is useful in distinguishing between these entities. As cases of peripheral T-cell lymphoma complicated by a proliferation of large B cells and angioimmunoblastic T-cell lymphoma with a large B-cell proliferation may exhibit overlapping morphologic features and likely have different biologic and survival characteristics, CD10 expression may serve as an additional useful discriminating criterion.

\section{Materials and methods}

\section{Case Selection}

A total of 33 cases consisting of peripheral T-cell lymphoma complicated by a large B-cell proliferation (10) angioimmunoblastic T-cell lymphoma (10) and angioimmunoblastic T-cell lymphoma with a large B-cell proliferation (13) were retrieved from the Stanford University Department of Pathology files during the period from 1996 to 2003. Approximately $90 \%$ of the cases were seen in consultation. The samples consisted of 32 lymph nodes and one lung biopsy.

\section{Morphologic Review}

Diagnoses were established according to the 2001 WHO criteria using a combination of morphologic, immunohistochemical and/or molecular findings. ${ }^{1}$ Seven of the cases of peripheral T-cell lymphoma complicated by a large B-cell proliferation have been previously reported. ${ }^{3}$ Angioimmunoblastic T-cell lymphoma was characterized by partial to complete effacement of the tissue architecture by atypical CD3 $(+)$ lymphoid cells with clear cytoplasm, increased vascularity, and CD21(+) follicular dendritic cells extending from high endothelial venules. As detected by CD20 immunohistochemistry, cases of angioimmunoblastic T-cell lymphoma with a large B-cell proliferation and peripheral T-cell lymphoma complicated by a large B-cell proliferation had an accompanying, increased population of large B cells.

\section{Immunohistochemistry}

Immunohistochemical analysis was performed on $5 \mu \mathrm{m}$ micron, formalin-fixed, paraffin-embedded sections in all cases. A broad immunohistochemical panel was utilized in the evaluation of the cases. Primary antibodies included CD3 (Dako, polyclonal), CD20 (Dako, clone L26), CD10 (Novocastra, New Castle-Upon-Tyne, UK, 1:40 dilution) and CD21 (Dako, clone IF8). CD10 positivity was assessed in each case by three pathologists (KKR, EJS and YN). Positivity was defined as discrete membrane staining in morphologically atypical, neoplastic cells. Dual staining for CD3 and CD10 was not able to be performed due to technical reasons. Statistical analysis was performed using a Fisher's exact test.

\section{In Situ Hybridization for Epstein-Barr Viral RNA}

In situ hybridization was performed on $5 \mu \mathrm{m}$, formalin-fixed, paraffin-embedded sections. Slides were stained with biotinylated Epstein-Barr Virus probe (Operon, Alameda, CA, USA). on an automated immunostainer (Ventana Benchmark, Tucson, AZ, USA). Specific nuclear signal was considered positive. An oligo dT to verify the presence of intact RNA within tumor cells in the tissue section and a known positive control were run with each case.

\section{Molecular Studies}

Polymerase chain reaction to evaluate B-cell immunoglobulin heavy chain and T-cell receptor gamma gene rearrangement was performed according to a previously described method. ${ }^{6}$

\section{Results}

\section{Clinical Features}

Selected clinical features from our 33 cases are depicted in Tables 1 and 2. Peripheral T-cell lymphoma complicated by a proliferation of large $\mathrm{B}$ cells and angioimmunoblastic T-cell lymphoma 
Table 1 Selected clinical, immunophenotypic and molecular features in peripheral T-cell lymphoma complicated by a large B-cell proliferation

\begin{tabular}{lllllllll}
\hline Case & Diagnosis & Sex & $\begin{array}{c}\text { Age } \\
\text { (years) }\end{array}$ & Biopsy site & $\begin{array}{l}\text { Gene } \\
\text { rearrangement }\end{array}$ & $\begin{array}{l}\text { CD10 } \\
\text { IHC }\end{array}$ & $\begin{array}{l}\text { CD21 expanded } \\
\text { follicular dendritic } \\
\text { cell meshworks }\end{array}$ \\
\hline in large B \\
cells
\end{tabular}

PTCL-LB, peripheral T-cell lymphoma complicated by a proliferation of large B cells; LN, lymph node; R, right; L, left; ND, not done; T, TCRgamma gene rearrangement; B, IgH gene rearrangement; EBV, Epstein-Barr virus in situ hybridization (EBER); (-/+), oligoclonal pattern.

Table 2 Selected clinical, immunophenotypic and molecular features in angioimmunoblastic T-cell lymphoma and angioimmunoblastic T-cell lymphoma with large B cells

\begin{tabular}{|c|c|c|c|c|c|c|c|c|}
\hline Case & Diagnosis & Sex & $\begin{array}{c}\text { Age } \\
\text { (years) }\end{array}$ & Biopsy site & $\begin{array}{l}\text { Gene } \\
\text { rearrangement }\end{array}$ & $\begin{array}{l}\text { CD10 } \\
\text { IHC }\end{array}$ & $\begin{array}{l}\text { CD21 expanded } \\
\text { follicular dendritic } \\
\text { cell meshworks }\end{array}$ & $\begin{array}{l}\text { EBV status } \\
\text { in large } B \\
\text { cells }\end{array}$ \\
\hline 1 & AITL & $\mathrm{F}$ & 51 & LN, cervical & $(+) \mathrm{T}$ & Pos & Pos/neg & \\
\hline 2 & AITL & $\mathrm{M}$ & 82 & LN, R ing & $(+) \mathrm{T}$ & Pos & Pos & \\
\hline 3 & AITL & $\mathrm{M}$ & 56 & LN, $R$ ing & ND & Pos & Pos & \\
\hline 4 & AITL & $\mathrm{F}$ & 78 & LN, periaortic & $(-) \mathrm{T}$ & Neg & Pos & \\
\hline 5 & AITL & $\mathrm{M}$ & 72 & LN, L neck & $(+) \mathrm{T}$ & Pos & Pos & \\
\hline 6 & AITL & $\mathrm{M}$ & 62 & LN, R inguinal & ND & Pos & ND & \\
\hline 7 & AITL & $\mathrm{F}$ & 52 & LN, L cervical & ND & Pos & ND & \\
\hline 8 & AITL & $\mathrm{F}$ & 54 & LN & ND & Pos & Pos & \\
\hline 9 & AITL & $\mathrm{M}$ & 52 & LN, L inguinal & $(+) \mathrm{T}$ & Pos & Pos & \\
\hline 10 & AITL & $\mathrm{F}$ & 65 & LN, R post thigh & ND & Pos & Pos & \\
\hline 11 & AITL-LB & $\mathrm{M}$ & 72 & LN, R inguinal & $(+) \mathrm{T},(-) \mathrm{B}$ & Neg & Pos & Pos \\
\hline 12 & AITL-LB & $\mathrm{F}$ & 76 & LN, R axillary & ND & Neg & Pos & ND \\
\hline 13 & AITL-LB & $\mathrm{M}$ & 73 & LN, L cervical & ND & Neg & Pos & Pos \\
\hline 14 & AITL-LB & $\mathrm{M}$ & 49 & LN, L axillary & $(+) \mathrm{T},(-) \mathrm{B}$ & Pos & Neg & Neg \\
\hline 15 & AITL-LB & $\mathrm{F}$ & 85 & LN, axillary & $(-) \mathrm{T},(+) \mathrm{B}$ & Neg & Pos & ND \\
\hline 16 & AITL-LB & $\mathrm{M}$ & 70 & LN, R inguinal & $(+) \mathrm{T},(-) \mathrm{B}$ & Pos & Pos & ND \\
\hline 17 & AITL-LB & $\mathrm{F}$ & 54 & LN, L cervical & $(+) \mathrm{T},(-) \mathrm{B}$ & Pos & Pos & Neg \\
\hline 18 & AITL-LB & $\mathrm{M}$ & 81 & LN, $\mathrm{R}$ inguinal & $(+) \mathrm{T},(-) \mathrm{B}$ & Neg & Pos & Pos \\
\hline 19 & AITL-LB & $\mathrm{M}$ & 64 & LN, L axillary & ND & Pos & Pos & Pos \\
\hline 20 & AITL-LB & M & 74 & LN, L neck & $(-) \mathrm{T},(-) \mathrm{B}$ & Pos & Pos & Pos \\
\hline 21 & AITL-LB & $\mathrm{M}$ & 76 & LN, cervical & ND & Pos & Pos & Pos \\
\hline 22 & AITL-LB & M & 54 & LN, cervical & ND & Pos & Pos & Pos \\
\hline 23 & AITL-LB & $\mathrm{M}$ & 68 & LN, R parotid & $(+) \mathrm{T},(+) \mathrm{B}$ & Pos & Pos & Neg \\
\hline
\end{tabular}

AITL, angioimmunoblastic T-cell lymphoma; AITL-LB, angioimmunoblastic T-cell lymphoma with large B cells; LN, lymph node; R, right; L, left; ND, not done; T, TCR-gamma gene rearrangement; B, IgH gene rearrangement; EBV, Epstein-Barr virus in situ hybridization (EBER); Pos/neg, only focally expanded dendritic cell network (akin to pattern 1 as described by Attygalle et $a l^{5}$ ).

with a large B-cell proliferation showed a male predominance (2-3:1) with mean age of 62 years (range 28-80) and 69 years (range 49-81), respectively. The 10 cases of angioimmunoblastic T-cell lymphoma demonstrated an equal sex distribution with a mean age of 62 years (range 51-82).

\section{CD10 Immunohistochemistry}

Eight of the 10 cases of peripheral T-cell lymphoma complicated by a proliferation of large $\mathrm{B}$ cells showed no CD10 staining by the neoplastic T cells (Figure 1). All cases had scattered positive cells serving as an internal control. Of the two positive cases, one was a lymph node biopsy which showed no morphologic or immunohistochemical features of angioimmunoblastic T-cell lymphoma (Figure 2). The second case, a lung biopsy from a patient with a history of treated, nodal based, diffuse large B-cell lymphoma, exhibited diffuse CD10 positivity among tumor cells.

Nine of the 10 cases of angioimmunoblastic T-cell lymphoma $(90 \%)$ and eight of the 13 cases of 


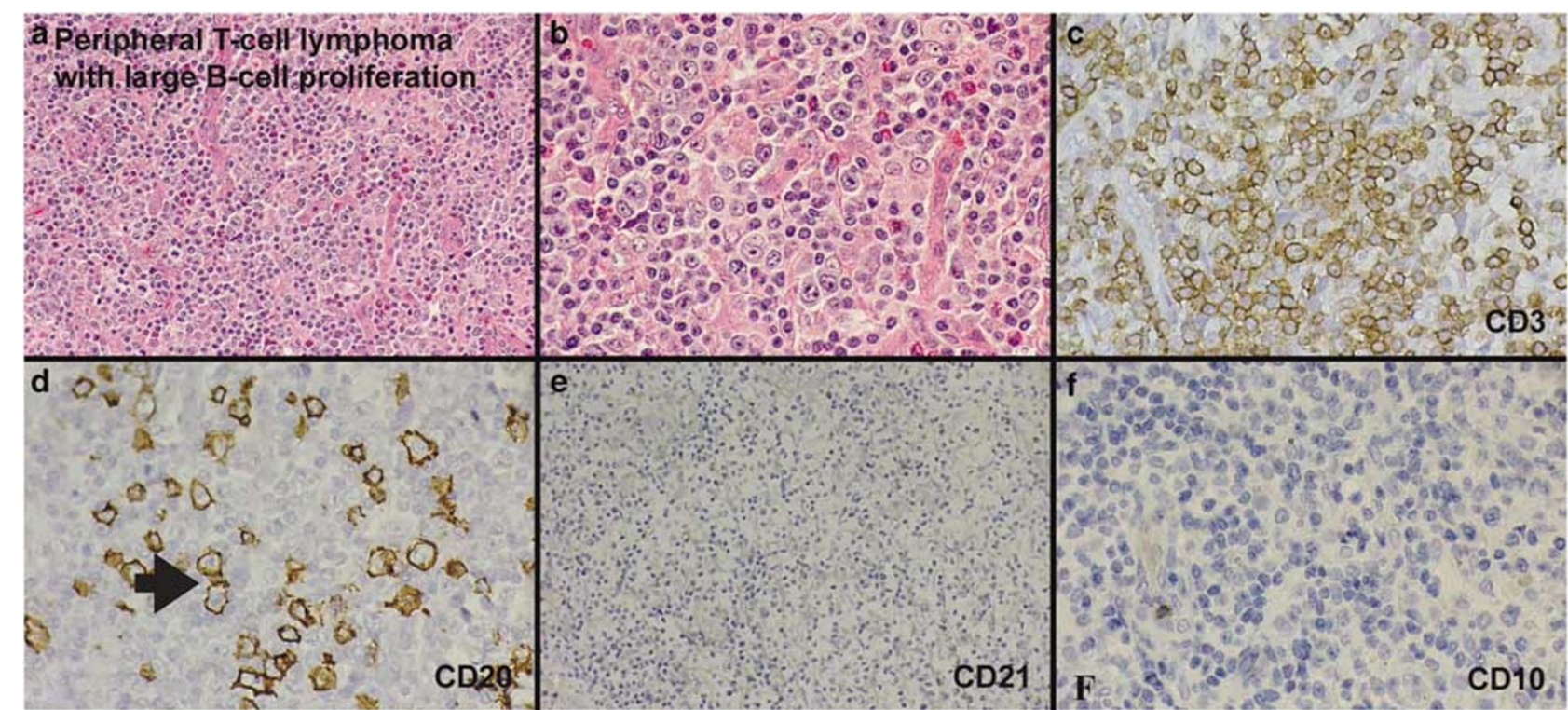

Figure 1 Peripheral T-cell lymphoma complicated by a proliferation of large B cells. (a and b) H\&E-stained sections, original magnification times $\times 40$ and $\times 400$, respectively. (c) CD3 immunostain. (d) CD20 immunostain highlighting the proliferation of large B cells. (e) CD21 showing lack of expanded follicular dendritic cell network. (f) CD10 immunostain showing lack of staining of neoplastic T cells.

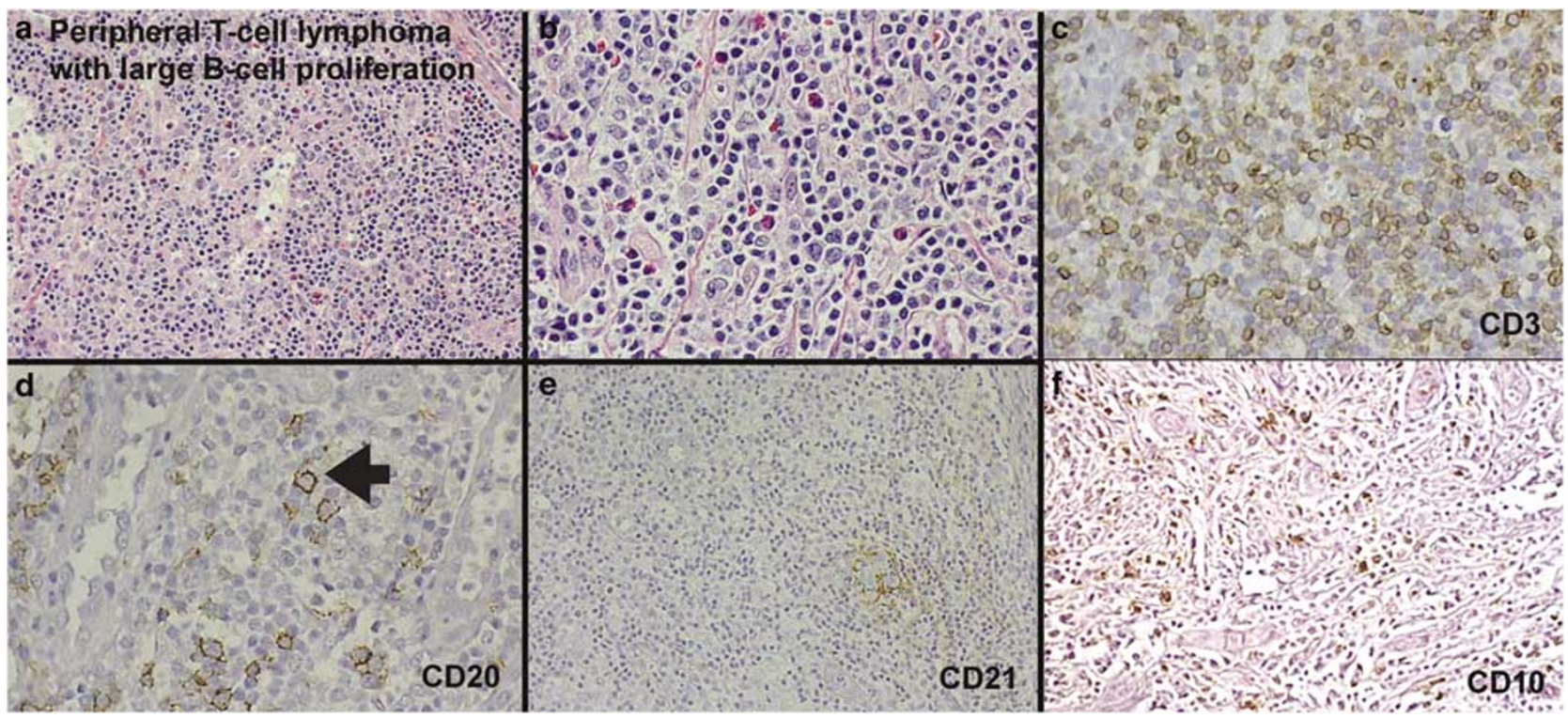

Figure 2 Peripheral T-cell lymphoma complicated by a proliferation of large B cells. (a and b) H\&E-stained sections, original magnification times $\times 40$ and $\times 400$, respectively. (c) CD3 immunostain. (d) CD20 immunostain highlighting the proliferation of large B cells. (e) CD21 showing minimal residual follicular dendritic cell network. (f) CD10 immunostain showing positive staining of neoplastic T cells.

angioimmunoblastic T-cell lymphoma with a large B-cell proliferation (62\%) showed CD10 expression by the malignant T cells. Representative morphology and selected immunohistochemistry from one case each of $\mathrm{CD} 10(+)$ angioimmunoblastic T-cell lymphoma and angioimmunoblastic T-cell lymphoma with a large B-cell proliferation are shown in Figures 3 and 4, respectively. The pattern of CD10 positivity in our cases was seen predominantly as an increased density of individual neoplastic cells throughout the involved tissue. Only one case showed clusters of positive malignant cells. The CD10-negative angioimmunoblastic T-cell lymphoma showed rare, CD10 $(+)$, atypical, small cells without unequivocal positivity in the neoplastic cells. This case had the typical morphologic (partial effacement of the 


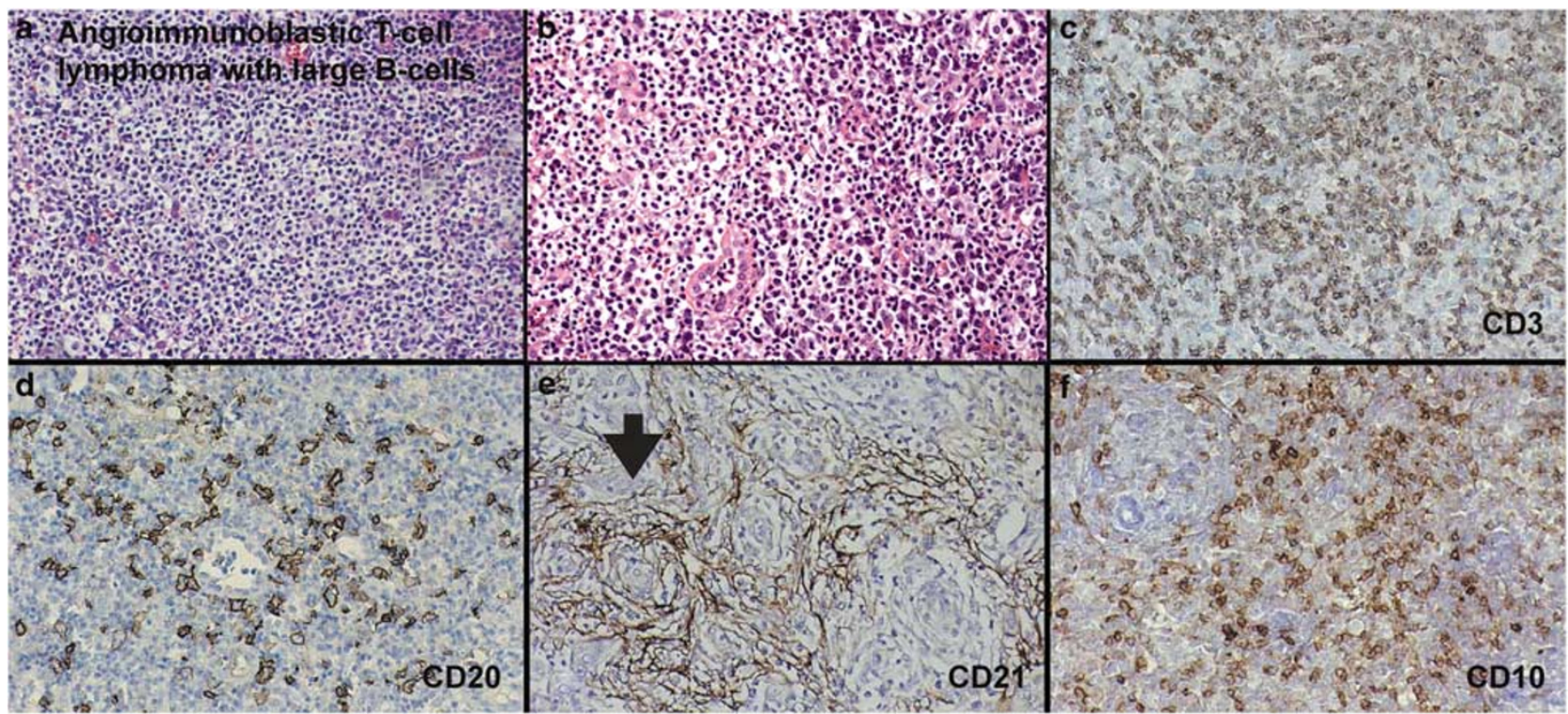

Figure 3 Angioimmunoblastic T-cell lymphoma with proliferation of large B cells. (a and b) H\&E-stained sections, original magnification times $\times 40$ and $\times 400$, respectively. (c) CD3 immunostain. (d) CD20 immunostain highlighting the proliferation of large B cells. (e) CD21 showing expanded and arborizing follicular dendritic cell networks. (f) CD10 immunostain showing positive staining of neoplastic T cells.

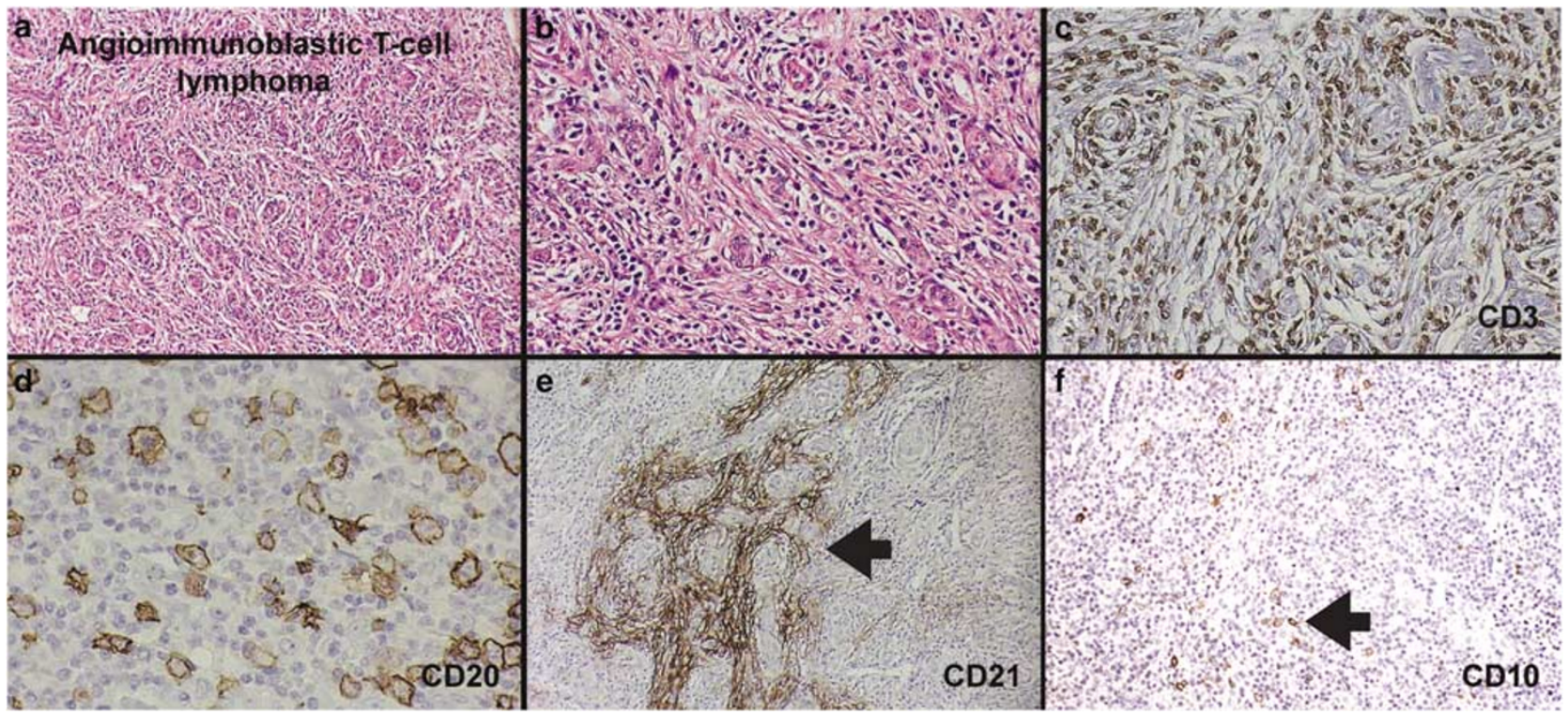

Figure 4 Angioimmunoblastic T-cell lymphoma. (a and b) H\&E-stained sections, original magnification times $\times 40$ and $\times 400$, respectively. (c) CD3 immunostain. (d) CD20 immunostain highlighting scattered large B cells. (e) CD21 showing expanded follicular dendritic cell networks. (f) CD10 immunostain showing positive staining of neoplastic T cells.

nodal architecture by atypical lymphoid cells with clear cytoplasm, increased vascularity) and immunohistochemistry $(\mathrm{CD} 21(+)$ follicular dendritic cells extending from high endothelial venules) features of angioimmunoblastic T-cell lymphoma. One case of angioimmunoblastic T-cell lymphoma with a large B-cell proliferation showed rare, slightly atypical, CD10 $(+)$ cells, and four cases were $\operatorname{CD} 10(-)$. These five cases exhibited the otherwise typical morphology and immunohistochemistry of angioimmunoblastic T-cell lymphoma (as described above). The finding of CD10 positivity in two of 10 cases of peripheral T-cell lymphoma complicated by a proliferation of large B cells vs eight of 13 cases of angioimmunoblastic T-cell lymphoma with a large B-cell proliferation is not statistically significant ( $P$-value of 0.057 , Fisher's exact test). 


\section{Molecular Studies}

Results of B-cell immunoglobulin heavy chain and/ or T-cell receptor-gamma gene rearrangement studies are displayed in Tables 1 and 2. As expected, most cases of angioimmunoblastic T-cell lymphoma, peripheral T-cell lymphoma complicated by a proliferation of large $B$ cells and angioimmunoblastic T-cell lymphoma with a large B-cell proliferation exhibited clonal T-cell receptor gene rearrangements where tested. In addition, two of eight cases of angioimmunoblastic T-cell lymphoma with a large B-cell proliferation and three of eight cases of peripheral T-cell lymphoma complicated by a proliferation of large B cells demonstrated B-cell immunoglobulin heavy chain gene rearrangements, highlighting the clonal nature of the accompanying $B$ cells in a subset of cases.

\section{Discussion}

In this study, we report the CD10 expression pattern in cases of peripheral T-cell lymphoma complicated by a proliferation of large B cells. Our findings reveal that only $20 \%$ of cases of peripheral T-cell lymphoma complicated by a proliferation of large B cells show CD10 expression in the neoplastic T cells compared to $90 \%$ of cases of angioimmunoblastic T-cell lymphoma and $62 \%$ of cases of angioimmunoblastic T-cell lymphoma with a large B-cell proliferation. Although this finding of $20 \mathrm{vs} 62 \%$ is not statistically significant ( $P$-value of 0.57 ), CD10, in conjunction with the usual morphologic and immunohistochemical markers, may serve as a useful discriminator in these cases. It may also be a particularly helpful tool when cases exhibit morphologic and/or immunohistochemical overlap with angioimmunoblastic T-cell lymphoma.

Peripheral T-cell lymphoma complicated by a proliferation of large B cells is a recently described entity characterized by a proliferation of large, often Epstein-Barr virus-infected, B cells in the background of peripheral T-cell lymphoma, unspecified. ${ }^{3}$ This large B-cell-rich T-cell lymphoma should not be mistaken for a B-cell lymphoproliferative disorder. The large B-cell proliferation is well described and has been seen not only in peripheral T-cell lymphoma, not otherwise specified, but also in angioimmunoblastic T-cell lymphoma., ${ }^{2,7}$ The presence of large cells varies but most authors require $>25 \%$ of the background cells to be large B cells. These B cells are often Epstein-Barr virus positive, although scattered Epstein-Barr virusinfected T cells have also been reported. ${ }^{2}$ We noted, in this series, that more often the large B cells were Epstein-Barr virus positive in angioimmunoblastic T-cell lymphoma with a large B-cell proliferation than peripheral T-cell lymphoma complicated by a proliferation of large B cells. This difference may be related to differences in the underlying immunosuppression state.
The B cells may show polyclonal, oligoclonal or monoclonal immunoglobulin heavy chain gene rearrangements. The latter finding raises the question of what we should call an immunoglobulin heavy chain gene rearrangement in the background of a peripheral T-cell lymphoma. Unless there are overt morphologic features (sheets of cells, architectural destruction) of a B-cell lymphoma, we characterize the lesion as peripheral T-cell lymphoma complicated by a proliferation of large B cells and note the accompanying immunoglobulin heavy chain gene rearrangement findings. It should be noted that rare cases of B-cell lymphoma after angioimmunoblastic T-cell lymphoma and concurrent angioimmunoblastic T-cell lymphoma and large B-cell lymphoma have been reported. ${ }^{8-11}$ Definitive classification of the B-cell process may be difficult, but it is most important not to overlook the underlying T-cell malignancy.

The significance of the accompanying large B cells has only been evaluated in a few cases, and therefore remains largely unknown. Lome-Maldonado et $a l^{4}$ have reported in a series of angioimmunoblastic Tcell lymphoma with a large B-cell proliferation that the presence of the large B cells made no significant prognostic impact. In their particular study, they proposed angioimmunoblastic T-cell lymphoma with a large B-cell proliferation as a specific subtype of angioimmunoblastic T-cell lymphoma, so that it would not be mistaken for a T-cell-rich large B-cell lymphoma.

Given that the most likely candidate(s) to be considered in the differential diagnosis of peripheral T-cell lymphoma complicated by a proliferation of large $\mathrm{B}$ cells is angioimmunoblastic T-cell lymphoma or angioimmunoblastic T-cell lymphoma with a large B-cell proliferation, our findings showing that cases of peripheral T-cell lymphoma complicated by a proliferation of large B cells lack aberrant CD10 expression are quite useful. In a recent hallmark article, Attygalle et $a l^{5}$ reported that the neoplastic T cells in angioimmunoblastic T-cell lymphoma show CD10 positivity in contrast to cases of PTCL, unspecified. The pattern of positivity is described as being within the residual follicle centers, around the follicles and then spilling out into the interfollicular areas. In our experience and in this study which includes 13 cases of angioimmunoblastic T-cell lymphoma, the pattern of CD10 positivity, though variable, is typically seen as increased numbers of cytologically neoplastic cells predominating in the areas away from regressed follicles and away from the vascular proliferation. The positivity, even without the benefit of dual CD3 and CD10 costaining, is a relatively straightforward assessment using a $\times 20$ objective.

In summary, we found that CD10 expression on neoplastic $\mathrm{T}$ cells is useful in the comparison of cases of peripheral T-cell lymphoma complicated by a proliferation of large $\mathrm{B}$ cells and angioimmunoblastic T-cell lymphoma with a large B-cell 
proliferation. The majority of cases of peripheral Tcell lymphoma complicated by a proliferation of large B cells $(80 \%)$ are negative for CD10 in contrast to $38 \%$ of cases of angioimmunoblastic T-cell lymphoma with a large B-cell proliferation. We think that this finding serves as an additional useful tool in the distinction of peripheral T-cell lymphoma complicated by a proliferation of large $\mathrm{B}$ cells and angioimmunoblastic T-cell lymphoma with a large B-cell proliferation, particularly in cases where the morphologic and/or immunohistochemical features seem to overlap. In addition, although the specific biology, therapy and survival data are better known and characterized for angioimmunoblastic T-cell lymphoma, it is likely that these data will be known for peripheral T-cell lymphoma complicated by a proliferation of large B cells in the future, and the importance of distinguishing these entities even greater.

\section{Acknowledgement}

This work was supported by Grant NIH CA 34233.

\section{References}

1 Jaffe ES, Harris NL, Stein H, et al. Pathology and Genetics of Tumors of Haematopoietic and Lymphoid Tissues. IARC Press: Lyon, 2001, pp 189-237.

2 Weiss LM, Jaffe ES, Liu XF, et al. Detection and localization of Epstein-Barr viral genomes in angioimmunoblastic lymphadenopathy and angioimmunoblastic lymphadenopathy-like lymphoma. Blood 1992; 79:1789-1795.
3 Higgins JPT, van de Rijn M, Jones CD, et al. Peripheral T-cell lymphoma complicated by a proliferation of large B cells. Am J Clin Pathol 2000;114:236-247.

4 Lome-Maldonado C, Canioni D, Hermine O, et al. Angioimmunoblastic T cell lymphoma (AILD-TL) rich in large B cells and associated with Epstein-Barr virus infection. A different subtype of AILD-TL? Leukemia 2002;16:2134-2141.

5 Attygalle AD, Al-Jehani R, Diss TC, et al. Neoplastic T cells in angioimmunoblastic T-cell lymphoma express CD10. Blood 2002;99:627-633.

6 Fodinger M, Buchmayer H, Schwarzinger I, et al. Multiplex PCR for rapid detection of T-cell receptorgamma chain gene rearrangements in patients with lymphoproliferative disease. Br J Haematol 1996;94: 136-139.

7 Anagnostopoulos I, Hummel M, Finn T, et al. Heterogeneous Epstein-Barr virus infection patterns in peripheral T-cell lymphoma of angioimmunoblastic lymphadenopathy type. Blood 1992;80:18041812.

8 Nathwani BN, Rappaport H, Moran EM, et al. Malignant lymphoma arising in angioimmunoblastic lymphadenopathy. Cancer 1978;41:578-606.

9 Abruzzo LV, Schmidt K, Weiss LM, et al. B-cell lymphoma after angioimmunoblastic lymphadenopathy: a case with oligoclonal gene rearrangements associated with Epstein-Barr virus. Blood 1993;82: 241-246.

$10 \mathrm{Xu} \mathrm{Y,} \mathrm{McKenna} \mathrm{RW,} \mathrm{Hoang} \mathrm{MP,} \mathrm{et} \mathrm{al.} \mathrm{Composite}$ angioimmunoblastic T-cell lymphoma and diffuse large B-cell lymphoma. Am J Clin Pathol 2002;118: 848-854.

11 Zettl A, Lee SS, Rudiger T, et al. Epstein-Barr Virusassociated B-cell lymphoproliferative disorders in angioimmunoblastic T-cell lymphoma and peripheral T-cell lymphoma, unspecified. Am J Clin Pathol 2002; $117: 368-379$. 